\title{
Limitations in Total Laparoscopic Hysterectomy in Bangladesh
}

\author{
Ila IJ ${ }^{1}$, Ghani $A^{2}$, Ferdouse $\mathrm{JA}^{3}$, Khatun $\mathrm{A}^{4}$, Rahman $\mathrm{S}^{5}$, Hasan $\mathrm{J}^{6}$, Hassan $\mathrm{MR}^{7}$
}

Conflict of Interest: None
Received: $13-02-2018$
Accepted: $08-05-2018$
www.banglajol.info/index.php/JSSMC

Key Words:

Laparoscopic hysterectomy, surgical risk factor, limitations in facilities.

\begin{abstract}
:
Laparoscopic Hysterectomy is achieving great popularity nowadays. Our people are becoming increasingly interested in new advances in this field of surgery. Highly skilled surgical technique, longer operative time and expensive technology are observed to be the factors that impede the advancements in laparoscopic surgery ${ }^{l}$.
\end{abstract}

Objective: The aim of our study is to analyze the limitations of laparoscopic hysterectomy in our country.

Methods: This is a retrospective observational study where we have reviewed demographic data, compliance of the patients, quality of anaesthesia, efficiency of supportive staffs, adequacy of appropriate instruments, continuous training of surgeons and surgical risk factors.

Total 80 Total Laparoscopic Hysterectomy (TLH) were done between January' 2016 to December'2017 in Shaheed Suhrawardy Medical College Hospital (ShSMCH), Dhaka.

Result: In this study, we analyzed eighty patients who underwent total laparoscopic hysterectomy for fibroid uterus, adenomyosis, recurrent post-menopausal bleeding, abnormal Uterine bleeding (AUB) etc. We tried to analyze some limitations and challenges which we can overcome. In spite of lack of appropriate instruments, adequate ICU support and repeated training in specialized centre at home and abroad, $87.5 \%$ patients recovered well.

The aim of our study was to identify the surgical risk factors of the patients and limitations in our theatre environment

[J Shaheed Suhrawardy Med Coll 2018; 10(1): 3-5] DOI: http://dx.doi.org/10.3329/jssmc.v10i1.38895

\section{Introduction:}

Hysterectomy is the most frequently performed major gynaecological surgical procedure in the case of abnormal uterine bleeding, fibroid uterus, post-menopausal bleeding, ovarian tumor of perimenopausal ladies ${ }^{2}$. Though there are three approaches in hysterectomy - abdominal,

1. Dr. Israt Jahan Ila, Associate Professor, Department of Obs. and Gynae, Shaheed Suhrawardy Medical College \& Hospital, Dhaka

2. Dr. Afroza Ghani, Associate Professor, Department of Obs. and Gynae, Shaheed Suhrawardy Medical College \& Hospital, Dhaka

3. Dr. Jannat Ara Ferdouse, Associate Professor, Department of Obs. and Gynae, Shaheed Suhrawardy Medical College \& Hospital, Dhaka

4. Dr. Asia Khatun, Associate Professor, Department of Obs. and Gynae, Shaheed Suhrawardy Medical College \& Hospital, Dhaka

5. Dr. Sumana Rahman, Junior Consultant, Department of Obstetrics and Gynaecology, Govt. Employees' Hospital, Fulbaria, Dhaka.

6. Dr. Jobayed Hasan, Assistant Registrar, Department of Cardiothoracic Surgery, NICVD, Dhaka

7. Dr. Mohammad Rashedul Hassan, Medical Officer, Department of Surgery, Shaheed Suhrawardy Medical College \& Hospital, Dhaka

Correspondence to: Dr. Dr. Israt Jahan Ila, Associate Professor, Department of Obs. and Gynae, Shaheed Suhrawardy Medical College \& Hospital, Dhaka vaginal, laparoscopic, $70-80 \%$ of all hysterectomies are performed abdominally. Laparoscopic hysterectomy, in its different forms, is an attractive and safe procedure for the management of benign gynaecological conditions and many authorities recommend its use on a larger extent. Laparoscopic hysterectomy (LH) has been introduced in 1989 by Harry Reich as an alternative to abdominal hysterectomy $(\mathrm{AH})$. Since then many studies have proved this technique feasible ${ }^{3}$. Laparoscopic hysterectomy has become an option for women and their surgeons to consider. There are many surgical advantages of laparoscopy particularly magnification of anatomy and pathology and access to the uterine vessels ${ }^{3,4}$. Several randomized trials have demonstrated the advantages of laparoscopic hysterectomy such as faster recovery, reduced hospital stay, less post-operative pain, less operative blood loss, lower infectious morbidity rate etc ${ }^{3}$.

\section{Materials and method:}

Sample size: A total of 80 patients were undergone TLH over a period of two years in a tertiary care hospital.

Methodology: This is a retrospective observational study. In our study we selected the patients of benign 
uterine pathology and $\leq 2$ previous abdominal operation.

In our study we did not include the patients with history of previous multiple operation ( $>2$ ), previous longitudinal abdominal incision, associated medical conditions such as cardiac disease, renal failure, hepatic failure etc.

Since we started performing this operation, we have collected necessary data from women. For each operation, the following data were systematically collected and entered into a database. The data are - Age, parity, body mass index, indication for TLH, caesarean section or adhesion causing surgery, surgeons experience, operating time, associated surgical procedure during TLH (Adhesiolysis, adnexectomy, myomectomy), uterine weight, hospital stay, recovery time, estimated blood loss, analgesic requirement and intraoperative complication, number of supportive staff, adequacy and capability of instruments. We also reviewed the workshop training attended by the consultants.

The data were tabulated in excel sheet and analysed using computer software SPSS version 17.

\section{Results:}

Table I

\begin{tabular}{lcc}
\multicolumn{3}{c}{ Indication of TLH } \\
Indication & $\mathrm{n}$ & $\%$ \\
\hline Fibroid Uterus & 27 & 33.75 \\
Adenomyosis & 12 & 15 \\
Endometriosis & 8 & 10 \\
AUB & 15 & 18.75 \\
CIN & 5 & 06.25 \\
OT & 8 & 10 \\
PID & 5 & 06.25 \\
& 80 & 100 \\
\hline
\end{tabular}

Among the indications, fibroid uterus was highest (33.75).

Table II

\begin{tabular}{lc}
\multicolumn{2}{c}{ Characteristics of 80 patients } \\
\hline Variables & Mean Values \\
\hline Age (in yr) & $45.2 \pm 8.1$ \\
Parity & $2.74 \pm 1.38$ \\
Uterine size (in gestational wk) & $9 \pm 3.5$ \\
Operation time (in min) & $100 \pm 30.5$ \\
Estimated blood loss (in ml) & $67 \pm 54$ \\
Requirement of analgesic (in mg) & $110 \pm 57$ \\
Length of hospital stay (in days) & $4 \pm 2$ \\
\hline
\end{tabular}

Of the 80 women who underwent TLH during the study period, the mean age $\pm \mathrm{SD}$ of patient was $45.2 \pm 8.1$ year, the mean parity was $2.74 \pm 1.38$

Table III

\section{Surgical risk factors}

Surgical risk factors

个BMI (>30) n 20

Previous multiple surgery $(\mathrm{H} / \mathrm{O} 2 \mathrm{C} / \mathrm{S})$ 10

Presence of adhesion - Grade IV endometriosis 04 Lateral or lower uterine segment fibroid $(>5 \mathrm{~cm}) \quad 02$

36

Total 36 patients had surgical risk factors, the rest 44 patients were apparently healthy

Table IV

\begin{tabular}{lcc}
\multicolumn{3}{c}{ Peri operative outcome } \\
Outcomes & $\mathrm{n}$ & $\%$ \\
\hline Good Perioperative outcome & 70 & 87.5 \\
Required conversion (Laparotomy) & 5 & 6.5 \\
Complications & 6.5 & \\
Bowel-bladder injury & 1 & \\
$\uparrow$ Haemorrhage & 2 & \\
Port site infection & 2 & \\
\hline
\end{tabular}

Among the patients, only $6.5 \%$ had complications

Table V

\begin{tabular}{lcc}
\multicolumn{3}{c}{ Supportive Staffs } \\
Supportive Staff & Available & Required \\
\hline Scrub nurse & 1 & 2 \\
Circulatory staff & 1 & 3 \\
Laparoscopy machine technician & 0 & 1 \\
Assistant Surgeon & 2 & 3 \\
\hline
\end{tabular}

Number of each level of supportive staffs were inadequate

Compliance of patient

Total number of counselled

patient $=200$

Number of patient

motivated $=80=40 \%$

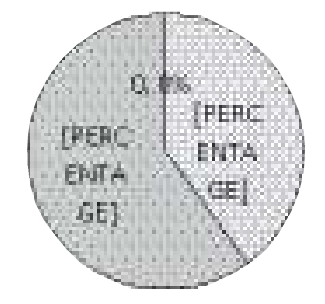

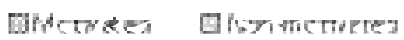


Training of the consultant

Total number of consultants $=42$

Number of consultants trained for the first time $=18$

Number of consultants frequently attending workshop, training, symposium $=10$

Number of consultants performing laparoscopy surgery independently $=6$

\section{Discussions}

Laparoscopic hysterectomy is currently accepted as a safe and efficient way to manage benign uterine pathology in selected patients. However, the challenges and limitation of this procedure are still debatable. The outcome of laparoscopic hysterectomy and the technique used in performing the operation well depend on various factors, such as the indication for hysterectomy, associated comorbidity, surgeon's experience and availability of the necessary equipment ${ }^{5}$.

Although laparoscopic surgery is well accepted by gynaecologist worldwide for the treatment of certain gynaecological conditions, laparoscopic hysterectomy in Germany, and probably world wide, is still only performed by few specialists ${ }^{6}$. Highly skilled surgical techniques, longer operating time and expensive technology are suggested to be the deterring factors ${ }^{5,6}$. Extensive experience in practicing and teaching various forms of laparoscopic hysterectomy(LH), laparoscopically vaginal hysterectomy(LAVH), total laparoscopic hysterectomy (TLH), classic intra fascial supra cervical hysterectomy(CISH) and laparoscopic supracervical hysterectomy( $\mathrm{LSH}$ ) have led to the firm conclusion that these techniques are advantageous to patients if performed for the appropriate indications ${ }^{7}$. Sixteen years have passed since the first publication of laparoscopy assisted vaginal hysterectomy, it is time to review and evaluate the limitations of laparoscopic hysterectomy. Although LH is well accepted, TLH is still only performed by a few specialists. In one study, only $40 \%$ patients want to do laparoscopic hysterectomy ${ }^{8}$.In our study, out of two hundreds councelled patients, eighty $(80)$ patients were undergone TLH, which $(40 \%)$ corresponds to the above study. Laparoscopic instruments(Harmonic scalpel, Vessel sealer, Bipolar diathermy, Ligasure etc) for TLH are expensive which are available in adequate amounts in only $40 \%$ cases in our institution though it is a tertiary level hospital. The rest $60 \%$ cases are done by conventional basic laparoscopic instruments. The experience of the laparoscopic surgeon is a crucial factor in defining the outcome ${ }^{5}$. Though the number of post graduate consultants are sufficient in our institution, among them skilled laparoscopic surgeons are few in number (14.28\%). The rest attempt their best however their efficiency is not up to par. This corresponds to another study where skilled surgeons are about $15 \%^{8}$. We have no technicians or trained nurses. There are many surgical risk factors like obese (25\%), having previous two caesarean section (12\%), having severe endometriosis $(3.2 \%)$.

In view of the aforementioned factors, our perioperative outcome was good $(87.5 \%$ cases $), 6.25 \%$ required conversion (laparotomy) and rest $6.25 \%$ had complications. Another study showed that even the most experienced surgeon was found to have a complication rate of 5.8$11.8 \%$ and a major complication rate of $2.2-2.7 \%$ after laparoscopic hysterectomy for benign pathology 9 . Our study corresponds to above study.

\section{Conclusion}

Laparoscopic hysterectomy is superior to conventional hysterectomy for surgeons in terms of well visualization of pelvic anatomy, ability to minimize blood loss, substantial and dynamic access to uterine vessels, vagina, rectum from many angles. For the patients it is less painful, less hospital stay but much expensive. Optimizing the operating theatre environment, adopting advanced instruments, enhancing surgeon's performance we can improve patient's outcome.

\section{References:}

1. Lee PI (1), Lee YT, Lee SH, Chang YK, “Advantages of Total Laparoscopic Hysterectomy”: (1)Jeil Women\&\#39; Hospital, Daechi-Dong 1021-4.

2. Mettler L1, Ahmed-Ebbiary N, Schollmeyer T, "Laparoscopic hysterectomy: challenges and limitations", Minim Invasive Ther Allied Technol. 2005;14(3):145-59.

3. A.L Gurin, AE. Kostiahin, D.O Kuchuk "Laparoscopic Hysterectomy: Advantages and disadvantages". Archives of perinatal medicine 18 (2), 110-112 January 2012.

4. Mel Kurtulus, MD, "Laparoscopic Hysterectomy: Many Advantages, but not widely offered; September 24, 2008.

5. M. Saleh, A. A. Seoud, M. S. Zaklama, Challenges of Laparoscopic hysterectomy: a 10-year experience in UK hospitals; Gynecological Surgery-May 2008, Vol 5, Issue 2, pp 115-120.

6. OBGYN. net staff, "overcoming Technical limits to Laparoscopic Hysterectomy” Oct 3, 2011.

7. A.L Gurin, A.E Kostianhin, D.O Kuchuk, "Laparoscopic Hysterectomy: Advantage and Disadvantages" Archives of Perinatal Medicine 18 (2): 110-112 January 2012.

8. Sara S Lange, MD, Laparoscopic Hysterectomy, Updated: Dec 24, 2015, https://emedicine. medscape.com/article/ 1839957.

9. Liu CY, Reich H (1994) Complications of total laparoscopic hysterectomy in 518 cases. Gynaecol Endosc 2:203-208. 